\title{
Optimization Design of the Ultra-High-Speed Vertical Rotor's Supporting Mechanism
}

\author{
Yongguang Liu, Xiaohui Gao, Xiaowei Yang, and Yixuan Wang \\ School of Automation Science and Electrical Engineering, Beihang University, Beijing 100191, China \\ Correspondence should be addressed to Xiaohui Gao; hgaoxiaohui@126.com
}

Received 18 December 2013; Accepted 23 January 2014; Published 31 March 2014

Academic Editor: Yunhua Li

Copyright (C) 2014 Yongguang Liu et al. This is an open access article distributed under the Creative Commons Attribution License, which permits unrestricted use, distribution, and reproduction in any medium, provided the original work is properly cited.

\begin{abstract}
How to increase the rotational speed and decrease vibration of the rotor in the acceleration has become an attractive subject, especially for the vertical rotors. This paper introduces a novel supporting mechanism to make the vertical rotor work at $80000 \mathrm{r} / \mathrm{min}$ smoothly. How to design and optimize the sensitive parameters of the supporting mechanism is the core problem to reduce the vibration in passing through critical speeds. Therefore, the FEM (finite element method) considering the gyroscopic couple is introduced to get the dynamic characteristic of the rotor system. The matching principle of the upper and lower supporting mechanism in the two-degree freedom system is extended to the multiple degree-freedom system, which is applied to optimize the parameters of the supporting mechanism combining with dynamic characteristic of the rotors system. At last, the rotor system can work at $80000 \mathrm{r} / \mathrm{min}$ smoothly in experiment.
\end{abstract}

\section{Introduction}

With the rapid development of manufacturing industry, the requirement of the rotate speed for the mechanical rotor is becoming more and more high, even to exceed the third or fourth order critical speed, and how to reach high speed and rotate smoothly is the core problems to be solved. When the rotational speed exceeds $20000 \mathrm{r} / \mathrm{min}$, the general supporting mechanism will not satisfy the requirement because of the influence of the strong centrifugal force, friction heat, or low-frequency eddy under a certain speed, which prevents the speed from continuing to rise. Especially for the vertical rotor, how to overcome the friction caused by gravity is a great puzzle. In the process of improving the speed of rotor, the severe instability and strong vibration will become more serious when it passes through the lower order critical speeds. Numbers of factors lead to the vibration of rotor system, such as the unbalance of rotor, the fault bearing, the nonuniform excitation, and the friction. In order to keep the stability of the rotor, the vibration attenuation will be one of the core problems in the design and applications. Therefore, we should pay more attention to the design of supporting mechanism. Since the traditional rotational supporting mechanism like bearings does not satisfy the requirements of ultra-highspeed rotor, some new mechanisms designed are presented in [1-5], which increase the speed through decreasing friction and adjusting damping in the rotation. In order to make rotor rotate smoothly, many experts invent the damper, shock absorber, and controller to reduce vibration [6-11], whose theory is regulating the damp and stiffness of the supporting system. Some papers introduce the application of the automatic dynamic balancer like ball balancer [12-15]. Although these supporting mechanisms make a contribution to improve the speed, they still cause the following problems: (1) continuing to raise the speed will become very difficult after reaching about $50000 \mathrm{r} / \mathrm{min}$; (2) although some rotor systems can reach $80000 \mathrm{r} / \mathrm{min}$, they are horizontal not vertical rotors; (3) they can produce the strong vibration and noise in the process of rising speed; (4) some experts have studied the supporting mechanism which is similar to this paper, but the parameter's selection of the supporting mechanism lacks sufficient theoretical support, which inhabits its development and application. In order to solve the above problems, we introduce a supporting mechanism and present the parameter's optimization to make the vertical rotor reach 80000 r/min smoothly. Firstly, we elaborate the structure and 
great superiority of this supporting mechanism in increasing the rotate speed. Then, the finite element model and matrixes are established considering the gyroscopic couple, which acquires dynamic characteristics of the rotor system. The principle of the supporting mechanism's parameter optimization method is proposed and the excellent effect of the dynamic response based on the above FEM is achieved in the fourth part of this paper. At last, the running and debugging experiment of the rotor system is carried out to guarantee that the rotor reaches $80000 \mathrm{r} / \mathrm{min}$ smoothly, which not only significantly shorten debugging cycles and promote its widespread application but also provide important reference for the design of ultra-high-speed vertical rotor's supporting mechanism.

\section{The Supporting Mechanism of Vertical Rotor}

This part introduces a supporting mechanism which is presented in Figure 1. The electromotor disk is a driving device connecting with the rotor, which is placed into alternating magnetic field. The rotor system can obtain arbitrary speed through adjusting the frequency of the magnetic field. The lower supporting mechanism is composed of the small shaft and damper. When the rotor reaches a certain speed, the oil film will be formed in the bottom of the small shaft to make rotor suspend, which greatly reduces the friction. As the smaller support stiffness of the lower supporting mechanism, the frequency response of the bottom supporting is very low and serious vibration of shaking head will appear in the relatively lower speed. In order to help the rotor pass the first and second order critical speed smoothly, the upper supporting mechanism is introduced. A ring shaped permanent magnet $(\mathrm{PM})$ is placed in the damper installed in the upper supporting mechanism and a ring iron is fixed in the top of the rotor, which will produce axial attractive power and radial magnetic coupling stiffness between them. The axial attractive power can alleviate the pressure of the rotor to reduce friction and the radial magnetic coupling stiffness can inhibit the transverse vibration.

This supporting mechanism can make the rotor reach higher speed in theory, because it can reduce the friction in the bottom of rotor significantly and provide damping and stiffness to suppress the vibration. But, when we do the experiment of rising speed, the rotor system appeared the low-frequency eddy and the speed cannot continue to be raised. It takes us one more month to debug the stiffness and damping of the upper and lower supporting mechanism to make the rotor reach $80000 \mathrm{r} / \mathrm{min}$. Although the rotor can be accelerated to higher speed, it still showed strong noise and vibration in the lower speed stage. In order to observe the vibrational state in the process of acceleration, we, respectively, set two monitoring points located in the top and middle of the rotor system represented in Figure 2. In order to understand the vibration directly, we only regard baseband signal in the vibration signal as the analyzing basis. Figure 3 shows that the rotor system caused strong vibration near the low order critical speed and tended to be stable as

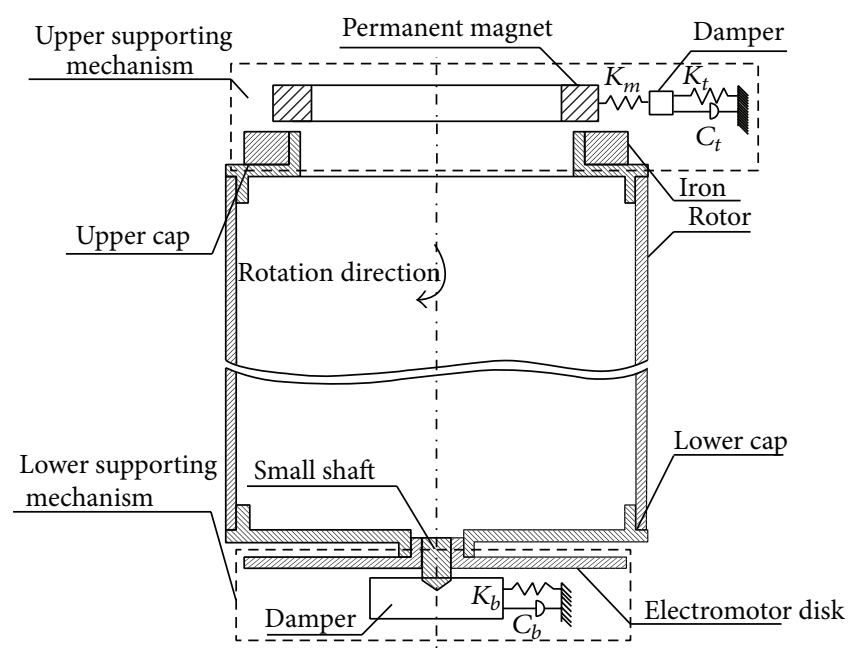

FIGURE 1: Structure of the rotor system. $K_{m}$ : radial magnetic coupling stiffness; $K_{t}$ : stiffness of the upper damper; $C_{t}$ : damping coefficient of the upper damper; $K_{b}$ : stiffness of the lower damper; $C_{b}$ : damping coefficient of the lower damper.

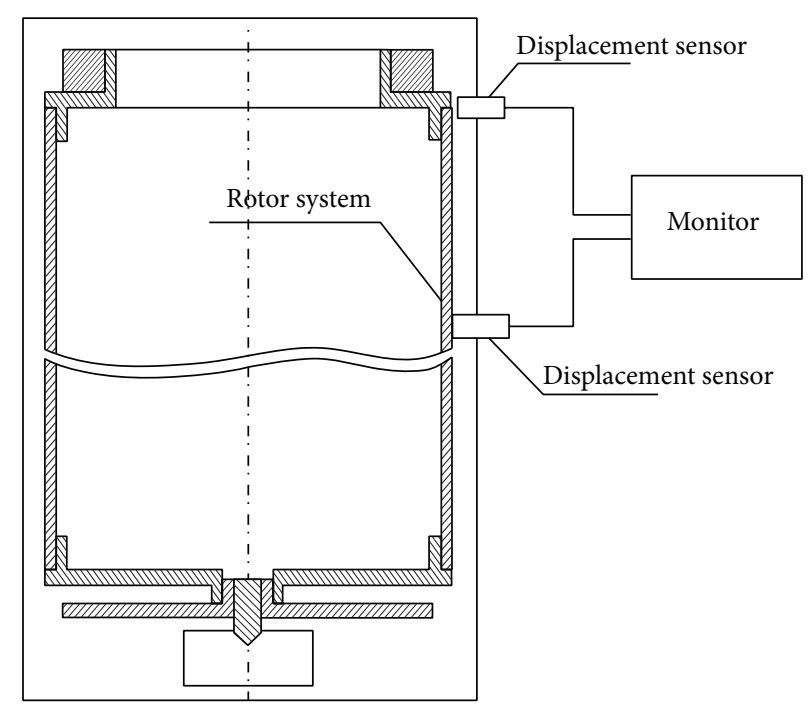

FIGURE 2: Vibration monitoring.

speed increases, which just catered to the strong noise in the initial phase. Therefore, matching of the upper and bottom supporting mechanism's parameter became the key factor to guarantee the rotor passing the critical speed smoothly.

\section{Dynamics Analysis of the Rotor System}

With the development of computer applications technology, finite element method (FEM) is applied to the engineering field very well and rotor dynamics analysis provides more accurate results by using FEM $[16,17]$. According to the specific situations, many studies develop different models to conduct the dynamics analysis in the foundation of Jeffcott rotor and achieve great performance. In this paper, we establish a new model to investigate the dynamic response of 


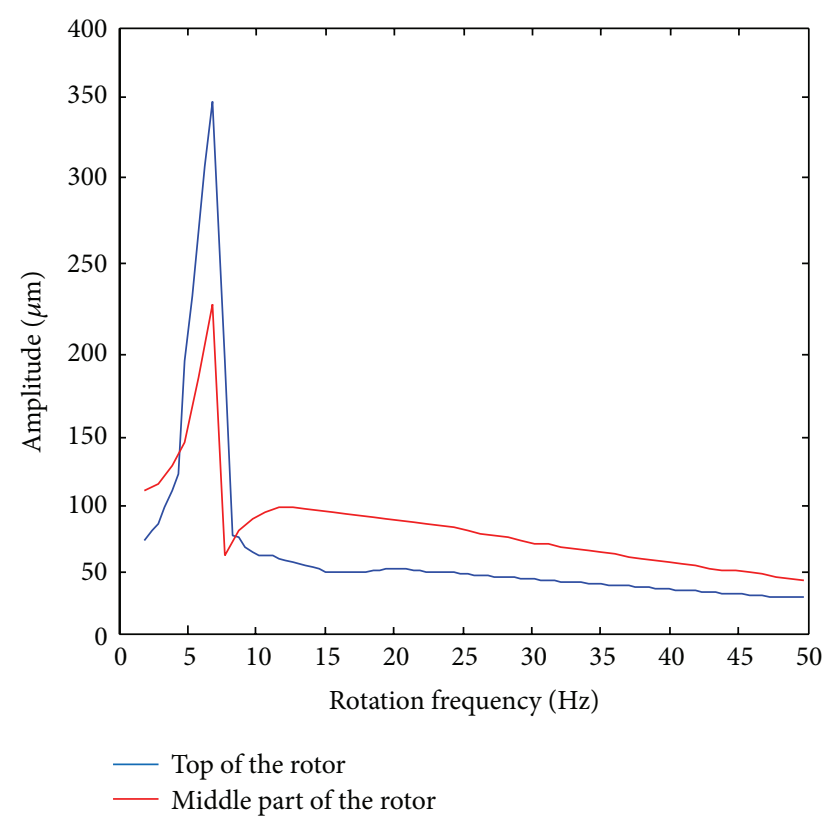

FIGURE 3: Response curves of the rotor.

the high speed rotor considering effects of gyroscopic couple on the basis of previous studies [18-21].

3.1. The Finite Element Modeling of the Rotor System. The finite element model of the rotor system is established in Figure 3. We divide the rotor into $n-1$ elastic elements of lumped mass which are linked through nodes without considering the three-dimensional specific shape. Firstly, we number all the nodes from top to bottom in the rotor system and build matrix for every node including mass matrix, stiffness matrix, damping matrix, and rotary matrix considering gyroscopic moment. Then, each unit matrix is stacked to the corresponding global matrix to get the global mass matrix, stiffness matrix, damping matrix, and rotary matrix and the dynamics differential equations of rotor system will be established with the force matrix of each node. At last, the linear algebraic equations are established through bringing the supposed steady-state solution into the differential equations and we can achieve the critical frequency and modal of the rotor system by solving them. There is one point needed to be explained; when the freedom degrees of element especially the boundary element are restrained, the element matrixes are not reduced for guaranteeing the generality of the calculation procedure and the boundary constraint method is applied after the formation of the global matrixes [22].

3.2. Establishing the Element Matrix of the Rotor System. There is a hypothesis that displacement column matrix of any node in the rotor system is $\left\{u_{1}\right\}=\left[\begin{array}{ll}x & \theta_{y}\end{array}\right]^{T}$ and $\left\{u_{2}\right\}=$ $\left[\begin{array}{ll}y & -\theta_{x}\end{array}\right]^{T}$. Then, each node matrix is $4 \times 4$ order submatrix and the global matrix with $\mathrm{n}$ nodes of the rotor system becomes the $2 n \times 2 n$ order sparse symmetric matrix. We divided the rotor into $n-1$ elements and the rotor has $n$ nodes. Element mass matrix, rotary matrix, and stiffness matrix of the rotor node are as follows [23]:

$$
\begin{aligned}
& {\left[M_{e t}\right]=\left[\begin{array}{cccc}
\frac{m}{2} & 0 & 0 & 0 \\
0 & 0 & 0 & 0 \\
0 & 0 & \frac{m}{2} & 0 \\
0 & 0 & 0 & 0
\end{array}\right]} \\
& {\left[M_{e R}\right]=\left[\begin{array}{cccc}
0 & 0 & 0 & 0 \\
0 & \frac{J_{d}}{2} & 0 & 0 \\
0 & 0 & 0 & 0 \\
0 & 0 & 0 & \frac{J_{d}}{2}
\end{array}\right]} \\
& {\left[M_{e}\right]=\left[M_{e t}\right]+\left[M_{e R}\right]=\left[\begin{array}{cccc}
\frac{m}{2} & 0 & 0 & 0 \\
0 & \frac{J_{d}}{2} & 0 & 0 \\
0 & 0 & \frac{m}{2} & 0 \\
0 & 0 & 0 & \frac{J_{d}}{2}
\end{array}\right] \text {, }} \\
& {\left[J_{e}\right]=\left[\begin{array}{cccc}
0 & 0 & 0 & 0 \\
& J_{p} & 0 & 0 \\
0 & \frac{2}{2} & 0 & 0 \\
0 & 0 & 0 & 0 \\
0 & 0 & 0 & \frac{J_{p}}{2}
\end{array}\right] \text {, }} \\
& =\frac{E I}{l^{3}(1+2 \nu)} \\
& \times\left[\begin{array}{cccc}
12 & 6 l & -12 & 6 l \\
6 l & 6 l^{2}-2 l^{2}(1-v) & -6 l & 2 l^{2}(1-\nu) \\
-12 & -6 l & 12 & -6 l \\
6 l & 2 l^{2}(1-\nu) & -6 l & 6 l^{2}-2 l^{2}(1-v)
\end{array}\right]
\end{aligned}
$$

$\left[K_{e}\right]$

$I$ : inertia moment of rotor's cross section; $m$ : mass of each rotor element; $l$ : length of each rotor element; $J_{p}$ : rotational inertia of each rotor element; $J_{d}$ : equatorial inertia moment of each rotor element; $v$ : Poisson's ratio of the rotor; E: elasticity modulus of the rotor.

Element matrixes of the node between upper damper and cap are as follows:

$$
\begin{gathered}
{\left[M_{e}^{1}\right]=\left[\begin{array}{cccc}
M_{t} & 0 & 0 & 0 \\
0 & 0 & 0 & 0 \\
0 & 0 & M_{1} & 0 \\
0 & 0 & 0 & J_{1}
\end{array}\right], \quad\left[J_{e}^{1}\right]=\left[\begin{array}{llll}
0 & 0 & 0 & 0 \\
0 & 0 & 0 & 0 \\
0 & 0 & 0 & 0 \\
0 & 0 & 0 & J_{1 p}
\end{array}\right],} \\
{\left[C_{e}^{1}\right]=\left[\begin{array}{cccc}
C_{t} & 0 & 0 & 0 \\
0 & 0 & 0 & 0 \\
0 & 0 & 0 & 0 \\
0 & 0 & 0 & 0
\end{array}\right],}
\end{gathered}
$$




$$
\left[K_{e}^{1}\right]=\left[\begin{array}{cccc}
K_{t}+K_{m} & 0 & -K_{m} & 0 \\
0 & 0 & 0 & 0 \\
-K_{m} & 0 & K_{m} & 0 \\
0 & 0 & 0 & 0
\end{array}\right] .
$$

$J_{1}$ : equatorial moment of inertia of the upper cap; $J_{1 p}$ : rotational inertia of upper cap.

Virtual joint section between upper cap and rotor is introduced and the element stiff matrix is as follows:

$$
\left[K_{e}^{2}\right]=\left[\begin{array}{cccc}
K_{\infty} & 0 & -K_{\infty} & 0 \\
0 & K_{1 h} & 0 & -K_{1 h} \\
-K_{\infty} & 0 & K_{\infty} & 0 \\
0 & -K_{1 h} & 0 & K_{1 h}
\end{array}\right] .
$$

$K_{\infty}$ is granted a greater value to guarantee consistency of the displacement between the upper cap and rotor and other matrixes not listed are null arrays.

Element matrixes of the node between the lower damper and rotor are as follows:

$$
\begin{gathered}
{\left[M_{e}^{n+2}\right]=\left[\begin{array}{cccc}
0 & 0 & 0 & 0 \\
0 & 0 & 0 & 0 \\
0 & 0 & M_{2} & 0 \\
0 & 0 & 0 & J_{2}
\end{array}\right], \quad\left[J_{e}^{n+2}\right]=\left[\begin{array}{llll}
0 & 0 & 0 & 0 \\
0 & 0 & 0 & 0 \\
0 & 0 & 0 & 0 \\
0 & 0 & 0 & J_{2 p}
\end{array}\right],} \\
{\left[K_{e}^{n+2}\right]=\left[\begin{array}{cccc}
K_{\infty} & 0 & -K_{\infty} & 0 \\
0 & K_{2 h} & 0 & -K_{2 h} \\
-K_{\infty} & 0 & K_{\infty} & 0 \\
0 & -K_{2 h} & 0 & K_{2 h}
\end{array}\right] .}
\end{gathered}
$$

$J_{2 p}$ : rotational inertia of the lower cap; $J_{2}$ : equatorial inertia moment of the lower cap; $K_{2 h}$ : angle stiffness of the lower cap.

$K_{\infty}$ is granted a greater value when calculating to guarantee consistency of the displacement between the lower cap and rotor and other matrixes not listed are null array.

Element matrix of the electromotor disk is as follows:

$$
\begin{aligned}
& {\left[M_{e}^{n+3}\right]=\left[\begin{array}{cccc}
0 & 0 & 0 & 0 \\
0 & 0 & 0 & 0 \\
0 & 0 & M_{4} & 0 \\
0 & 0 & 0 & J_{4}
\end{array}\right], \quad\left[J_{e}^{n+3}\right]=\left[\begin{array}{llll}
0 & 0 & 0 & 0 \\
0 & 0 & 0 & 0 \\
0 & 0 & 0 & 0 \\
0 & 0 & 0 & J_{4 p}
\end{array}\right],} \\
& {\left[K_{e}^{n+3}\right]} \\
& =\frac{E_{m} I_{m}}{l_{m}^{3}\left(1+2 v_{m}\right)} \\
& \quad \times\left[\begin{array}{cccc}
12 & 6 l_{m} & -12 & 6 l_{m} \\
6 l_{m} & 6 l_{m}^{2}-2 l_{m}^{2}\left(1-v_{m}\right) & -6 l_{m} & 2 l_{m}^{2}\left(1-v_{m}\right) \\
-12 & -6 l_{m} & 12 & -6 l_{m} \\
6 l_{m} & 2 l_{m}^{2}\left(1-v_{m}\right) & -6 l_{m} & 6 l^{2}-2 l^{2}\left(1-v_{m}\right)
\end{array}\right] .
\end{aligned}
$$

$M_{4}$ : mass of the electromotor disk; $J_{4}$ : rotational inertia of the electromotor disk; $J_{4 p}$ : equatorial moment of inertia of the electromotor disk; $I_{m}$ : rotational inertia of the electromotor disk; $E_{m}$ : elasticity modulus of the electromotor disk; $l_{m}$ : length of the electromotor disk; $v_{m}$ : Poisson's ratio of the electromotor disk.
Element stiffness matrix of the small shaft not considering mass is as follows:

$$
\left[K_{e}^{n+4}\right]=\frac{E_{r} I_{r}}{l_{r}^{3}}\left[\begin{array}{cccc}
12 & 6 l_{r} & -12 & 6 l_{r} \\
6 l_{r} & 4 l_{r}^{2} & -6 l_{r} & 2 l_{r}^{2} \\
-12 & -6 l_{r} & 12 & -6 l_{r} \\
6 l_{r} & 2 l_{r}^{2} & -6 l_{r} & 4 l_{r}^{2}
\end{array}\right] .
$$

$E_{r}$ : elasticity modulus of the small shaft; $I_{r}$ : inertia moment of the small shaft; $l_{r}$ : length of the small shaft.

In order to reflect the connecting boundary conditions between the small shaft and the lower damper, it is necessary to introduce the connecting virtual element. The displacement stiffness of the virtual element is infinite and the angular rigidity is zero, which is better than only considering the lateral stiffness of the small shaft. The stiffness matrix of the virtual element between small shaft and lower damper is as follows:

$$
\left[K_{e}^{n+5}\right]=\left[\begin{array}{cccc}
K_{\infty} & 0 & -K_{\infty} & 0 \\
0 & 0 & 0 & 0 \\
-K_{\infty} & 0 & K_{\infty} & 0 \\
0 & 0 & 0 & 0
\end{array}\right]
$$

Element matrix of the lower damper is as follows:

$$
\begin{gathered}
{\left[M_{e}^{n+6}\right]=\left[\begin{array}{cccc}
M_{b} & 0 & 0 & 0 \\
0 & 0 & 0 & 0 \\
0 & 0 & 0 & 0 \\
0 & 0 & 0 & 0
\end{array}\right], \quad\left[C_{e}^{n+6}\right]=\left[\begin{array}{cccc}
C_{b} & 0 & 0 & 0 \\
0 & 0 & 0 & 0 \\
0 & 0 & 0 & 0 \\
0 & 0 & 0 & 0
\end{array}\right],} \\
{\left[K_{e}^{n+6}\right]=\left[\begin{array}{cccc}
K_{b} & 0 & -K_{b} & 0 \\
0 & 0 & 0 & 0 \\
-K_{b} & 0 & K_{b} & 0 \\
0 & 0 & 0 & 0
\end{array}\right] .}
\end{gathered}
$$

3.3. To Solve the Differential Equation of the Rotor System. The rotor system has $n+7$ nodes in total according to the above methods and the global mass matrix, stiffness matrix, damp matrix, and rotary matrix are $2(n+7) \times 2(n+7)$ order symmetric sparse banded matrix whose half bandwidth is four. The displacement vectors of the rotor system are as follows:

$$
\begin{aligned}
& \left\{U_{1}\right\}=\left[x_{1}, \theta_{y 1}, x_{2}, \theta_{y 2}, \ldots, x_{n+7}, \theta_{y(n+7)}\right]^{T}, \\
& \left\{U_{2}\right\}=\left[y_{1},-\theta_{x 1}, y_{2},-\theta_{x 2}, \ldots, y_{n+7},-\theta_{x(n+7)}\right]^{T} .
\end{aligned}
$$

The differential equations of the rotor system are as follows [23]:

$$
\begin{aligned}
& {[M]\left\{\ddot{U}_{1}\right\}+[C]\left\{\dot{U}_{1}\right\}+\Omega[J]\left\{\dot{U}_{2}\right\}+[K]\left\{U_{1}\right\}=\left\{Q_{1}\right\},} \\
& {[M]\left\{\ddot{U}_{1}\right\}+[C]\left\{\dot{U}_{2}\right\}-\Omega[J]\left\{\dot{U}_{1}\right\}+[K]\left\{U_{2}\right\}=\left\{Q_{2}\right\} .}
\end{aligned}
$$

$[M],[C],[J]$, and $[K]$ are, respectively, standing for the global mass matrix, stiffness matrix, damp matrix, and rotary matrix which are expanded from the element matrix of every node according to the numbers. We will introduce the form 
of the global matrix to regard the form of the global stiffness matrix as example.

Consider

[K]

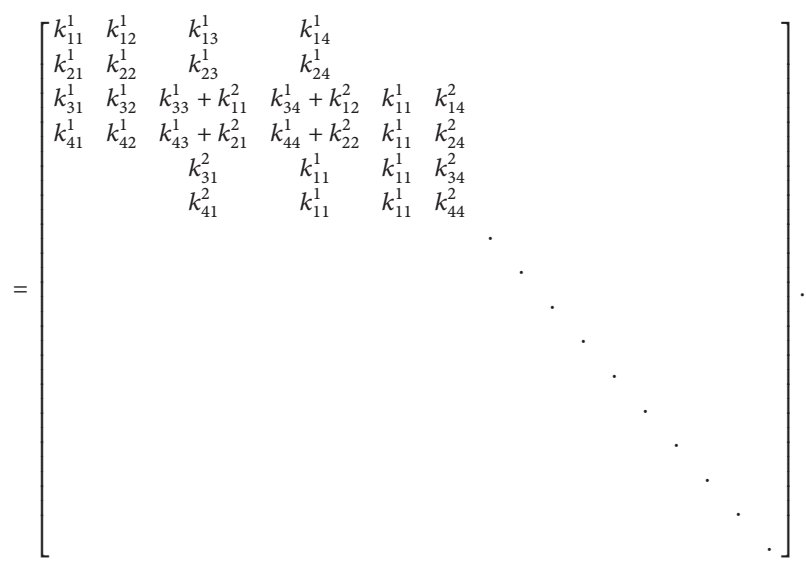

(11)

$k_{i j}^{n}(i=1,2,3,4 ; j=1,2,3,4)$ is an element at $i$ row and $j$ line of the stiffness matrix numbered $n$ and we can directly add it to the global stiffness matrix at row $2 n+i-2$ and $2 n+j-2$ line. The global mass matrix, damp matrix, and rotary matrix will be attained adopting the same method.

In order to simplify the calculation, we introduce $\{z\}=$ $\left\{u_{1}\right\}+i\left\{u_{2}\right\}$ and (10) will be transformed to plural equation:

$$
[M]\{\ddot{z}\}+[C]\{\dot{z}\}-i \Omega[J]\{\dot{z}\}+[K]\{z\}=\{Q\} .
$$

If you want to achieve the unbalance response, we can assume

$$
\{Q\}=\Omega^{2}\left[m_{1} e_{1} e^{i \phi_{1}}, 0, m_{2} e_{2} e^{i \phi_{2}}, 0, \ldots, m_{N} e_{N} e^{i \phi_{N}}, 0\right]^{T} e^{i \Omega t}
$$

and $m_{i}, e_{i}, \phi_{i}, \Omega(i=1,2,3, \ldots, N)$, respectively, represent the mass, eccentricity, phase angle of the nodes, and spin angular velocity of the rotor.

We assume that particular solution of the unbalance response is

$$
\{z\}=\{A\} e^{i \Omega t}
$$

and $\{A\}$ represents the plural column matrix of the steadystate response. We combine (14) with (12) to gain

$$
\begin{gathered}
{\left[-M \Omega^{2}+i \Omega C+J \Omega^{2}+K\right]\{A\}=\{F\}} \\
\left|-M \omega^{2}+i \omega C+J \omega \Omega+K\right|=0
\end{gathered}
$$

and $\{F\}=\Omega^{2}\left[m_{1} e_{1} e^{i \phi_{1}}, 0, m_{2} e_{2} e^{i \phi_{2}}, 0, \ldots, m_{N} e_{N} e^{i \phi_{N}}, 0\right]^{T}$ represents unbalanced force vector of the nodes.

We can get the principal mode and critical speed of any order through calculating the eigenvalue of (16) [24]. If we want to get the critical speed, we can assume $\Omega= \pm \omega$.

In order to greatly understand the characteristic of the rotor dynamics especially after considering gyroscopic couple, we mainly reveal the dynamic rules of the rotor system

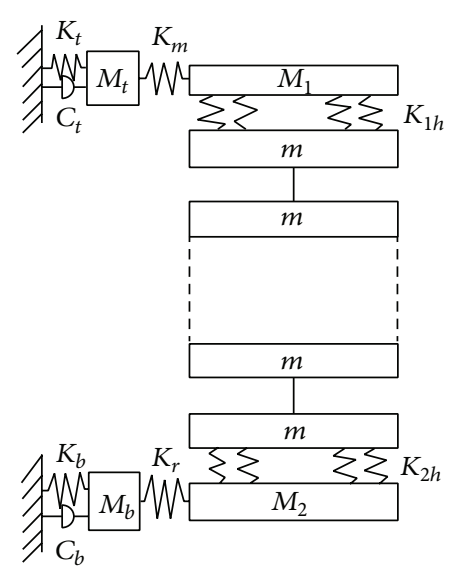

FIgURE 4: FEM model. $M_{t}$ : mass of the upper damper; $M_{1}$ : mass of the upper cap; $K_{1 h}$ : angular rigidity of the upper cap; $m$ : element mass; $K_{2 h}$ : angular rigidity of the lower cap; $K_{r}$ : stiffness of the small shaft; $M_{2}$ : mass of the lower cap.

through modal and unbalance response analysis and provide the theory data for the parameter selection of the damper.

We can get critical frequency synchronous forward precession and principal mode (Figure 4) of any orders through solving (16) after introducing the practical parameters of the rotor system without damp.

As is shown in Figure 5, the first order principal mode mainly displays swing of the top rotor, the second order displays swing of the top and bottom rotor simultaneously, the third order mainly displays the vibration of the upper damper, the forth order displays swing of the bottom rotor, and the fifth and sixth order principal mode mainly displays flexural vibrations of the rotor. In order to achieve $80000 \mathrm{r} / \mathrm{min}$, the rotor system must pass through the first four-order critical speed smoothly and steadily, and then how to select the parameter of the supporting mechanism will be the core problem of the study.

\section{Parameter's Optimization of the Supporting System}

The parameter's selection and matching of the upper and bottom supporting mechanism play a powerful role to guarantee the rotor rotating smoothly and the regulation of main parameters relies on selecting suitable damp and stiffness of the damper and magnetic coupling stiffness $K_{m}$. The damper is to restrain the vibration produced by all kinds of interference, so the amplitude of the rotor system attenuates down quickly to achieve stable running status. In practice, the upper and lower damper constitutes the avoiding vibration system which has two key parameters. One is optimal frequency ratio and the other is optimal damp. The specific optimization method is as follows: (1) we need to hunt for optimal frequency ratio based on one order mode; (2) we need to regulate the damp of the damper to achieve the optimum. There are two requirements to judge the optimizing parameters of the avoiding vibration system 


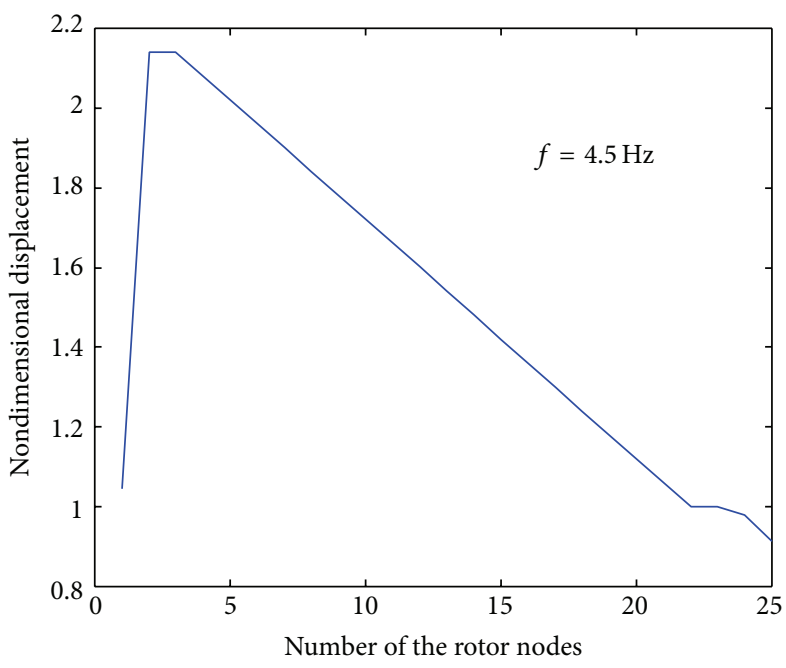

(a) The first order principal mode

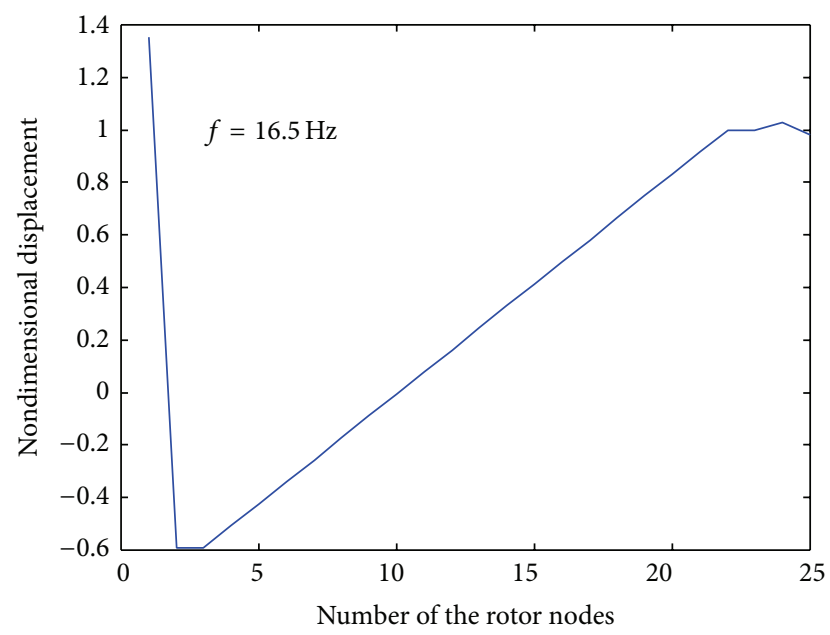

(c) The third order principal mode

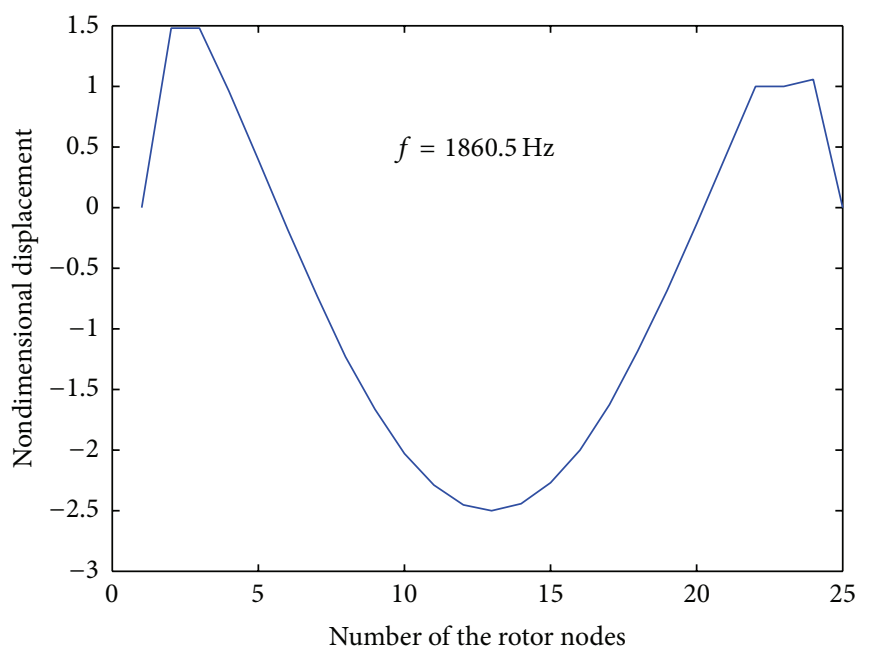

(e) The fifth order principal mode

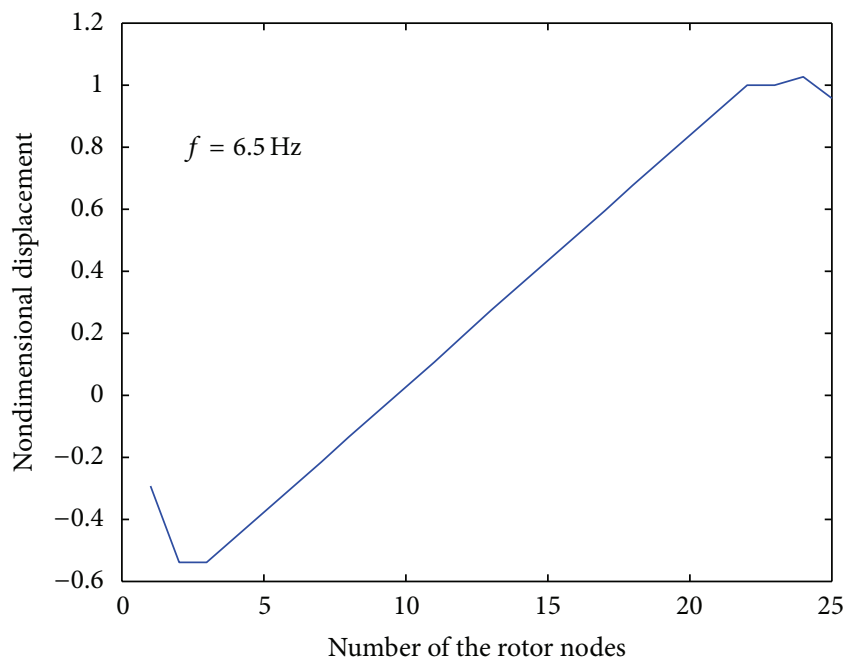

(b) The second order principal mode

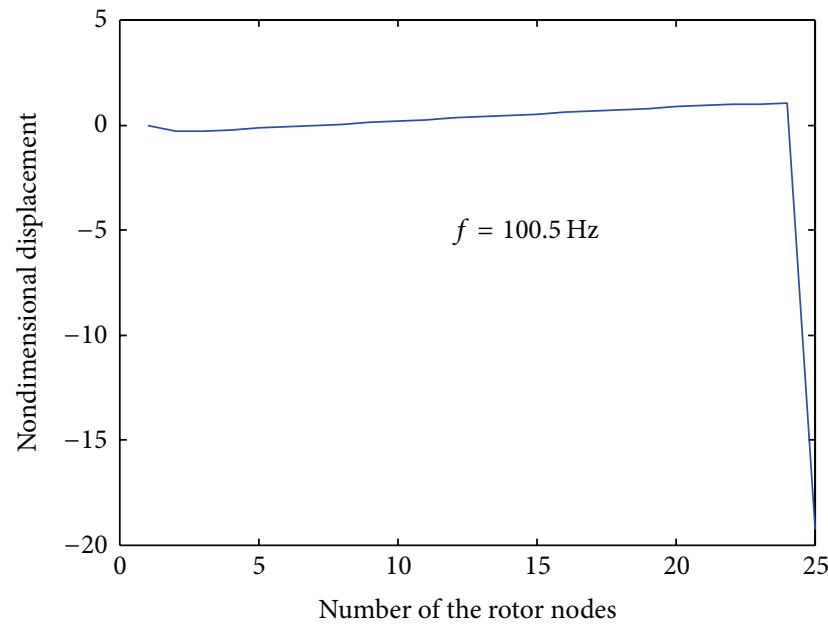

(d) The fourth order principal mode

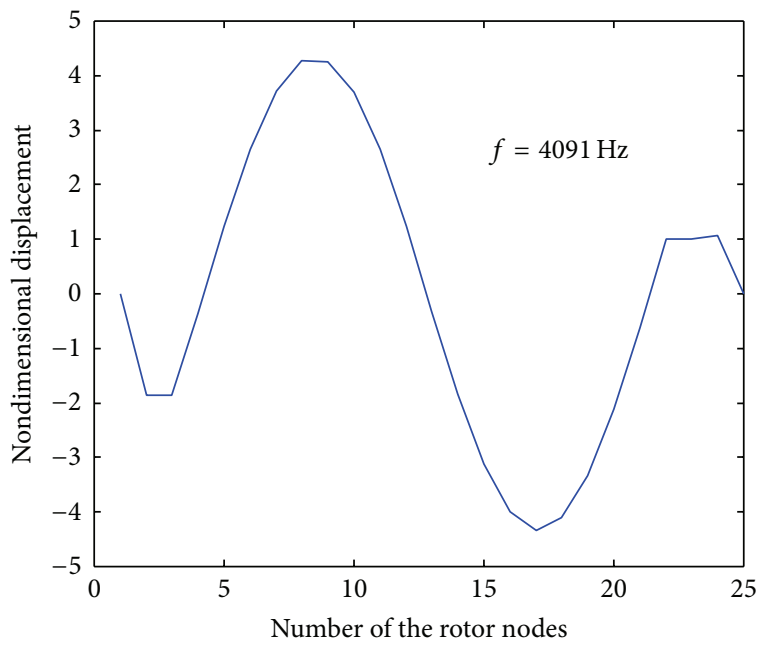

(f) The sixth order principal mode

Figure 5: Principal mode of the rotor. 


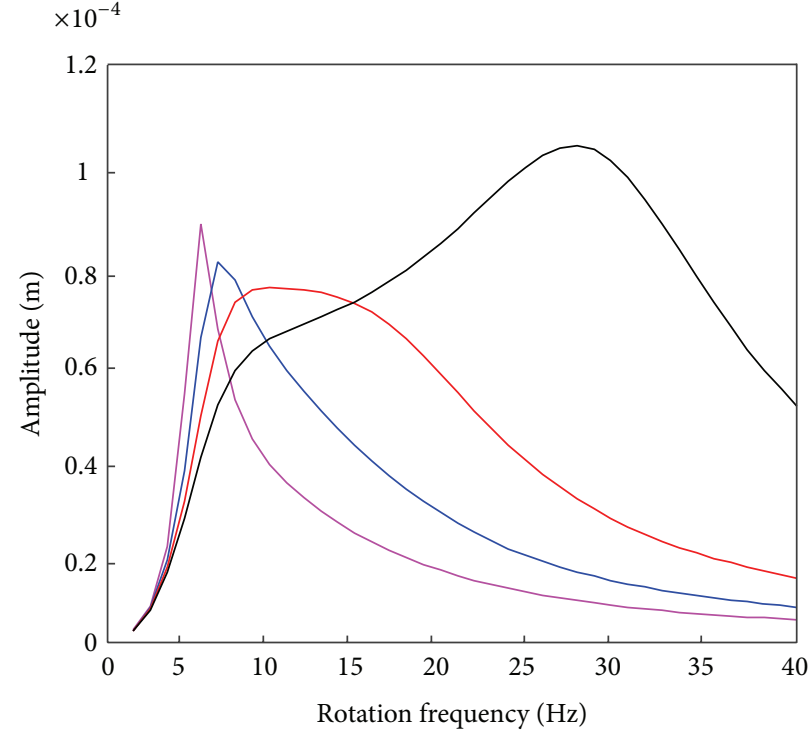

(a) Response of the upper damper

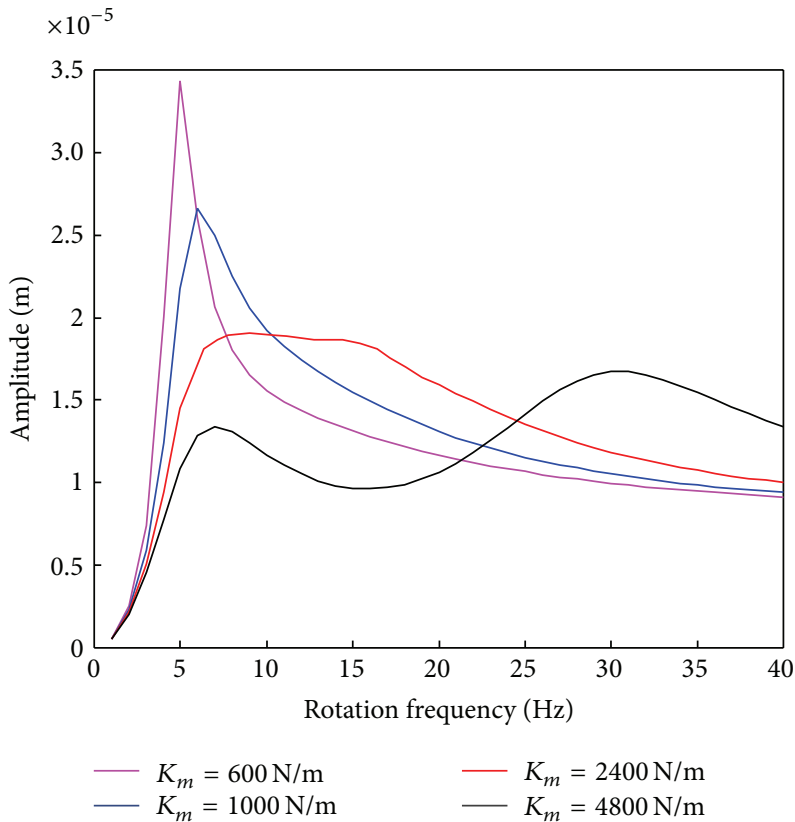

(c) Response of the middle rotor

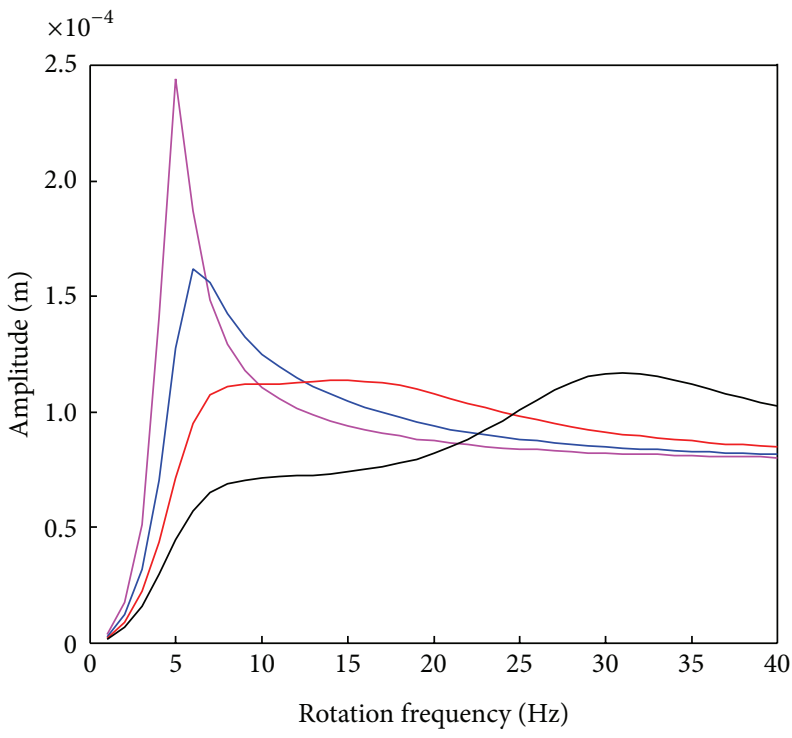

(b) Response of the top rotor

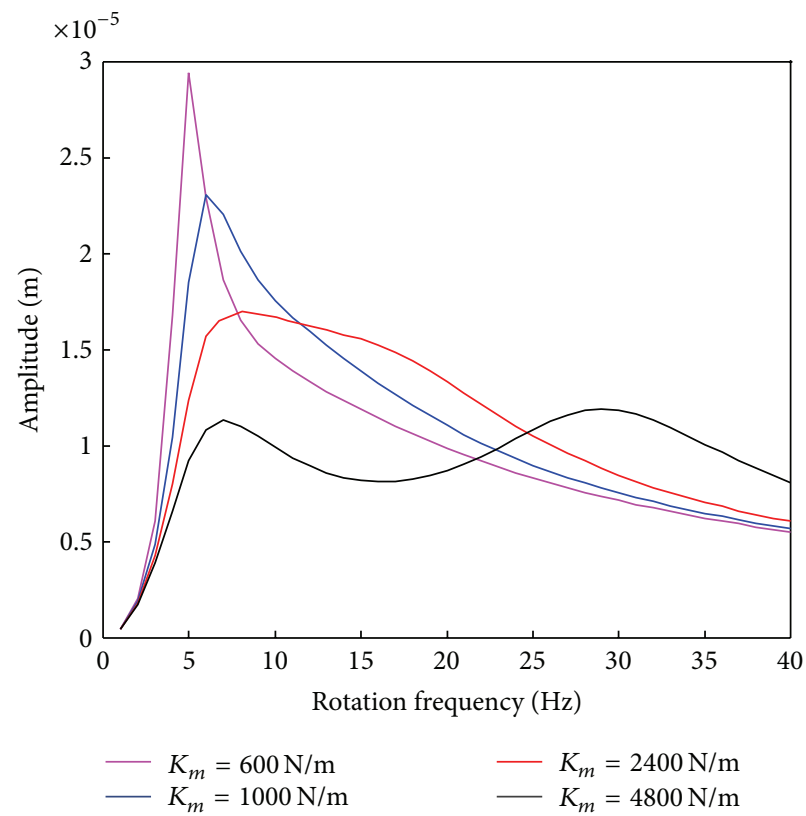

(d) Response of the lower damper

FIGURE 6: Response in different magnetic coupling stiffness.

according to the dynamic damping theory. (1) The two public points of the response curve at the spectrum chart adopting different damp have the same amplitude. (2) The peak of one response curve needs to be equal to the public point. It is clear that the public point is not related to the damp, so we need to change the public point only through regulating the frequency to make the two public points have the same amplitude which is named frequency modulation. Damping optimization is to achieve the coincidence point of the peak with the public point by regulating damp. These two processes can be regulated separately. Although this conclusion is achieved by damping system of two-degree freedom, it is adaptable to the multidegree freedom like the rotor system. The difference between the above two conditions is that the analytical expression of the optimal frequency ratio and damp can be attained more easily for the two-degree freedom system than for the multidegree freedom system. This study does not intend to deduce the complex formula than optimize structural parameters of the rotor system based on the above analysis.

The optimizing parameters steps of the rotor system are as follows.

(1) We need to confirm the modal to be tuned, estimate the frequency of the damper based on (17), and adjust the 


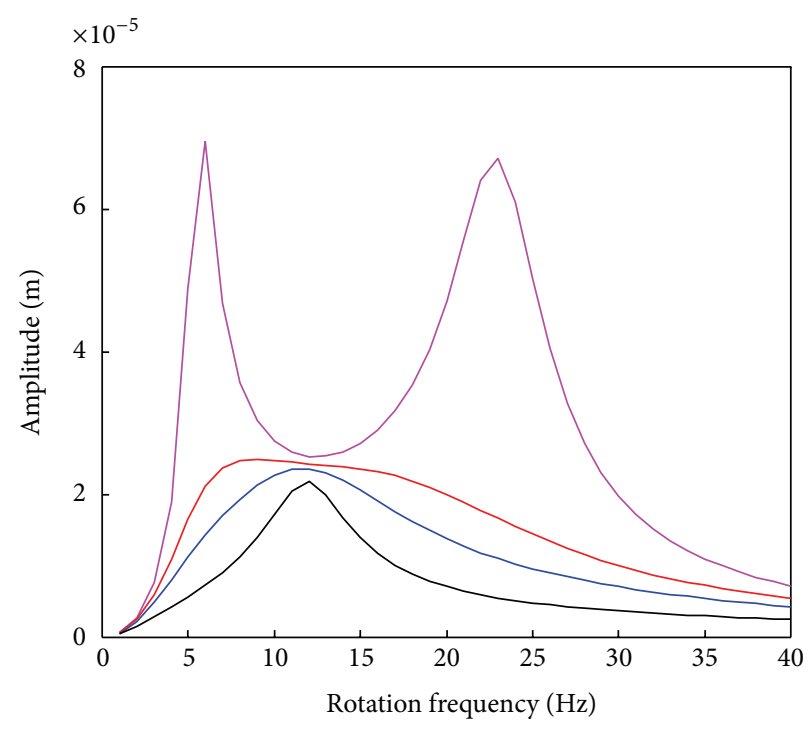

(a) Response of the upper damper

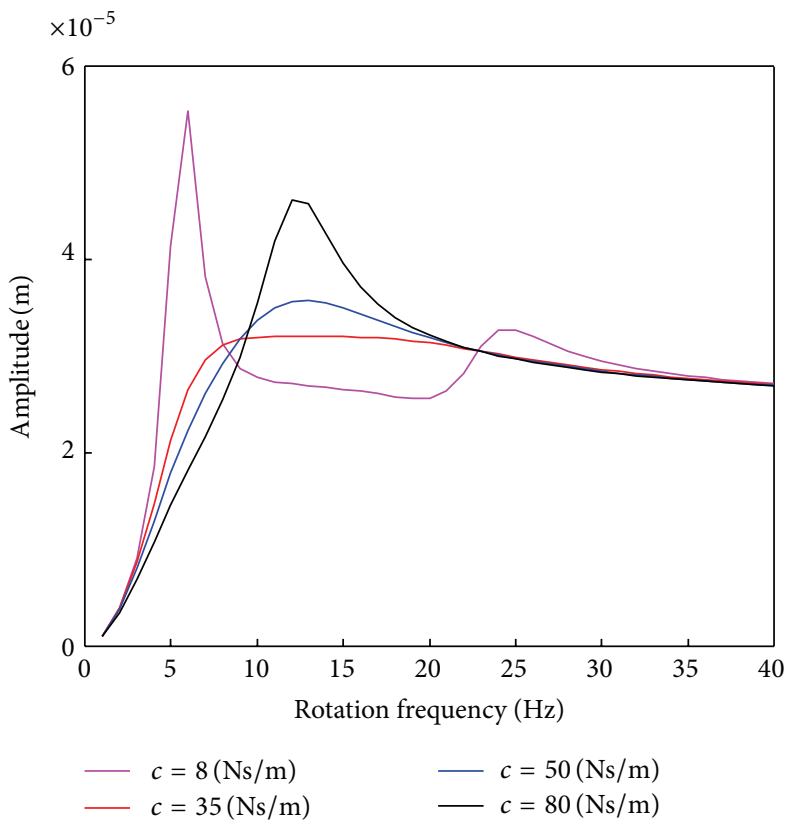

(c) Response of the middle rotor

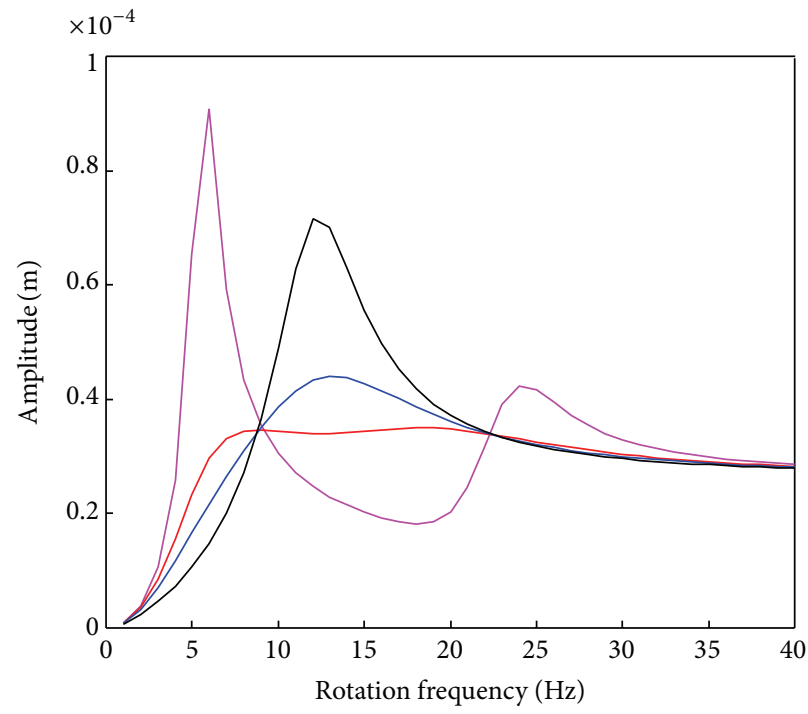

(b) Response of the top rotor

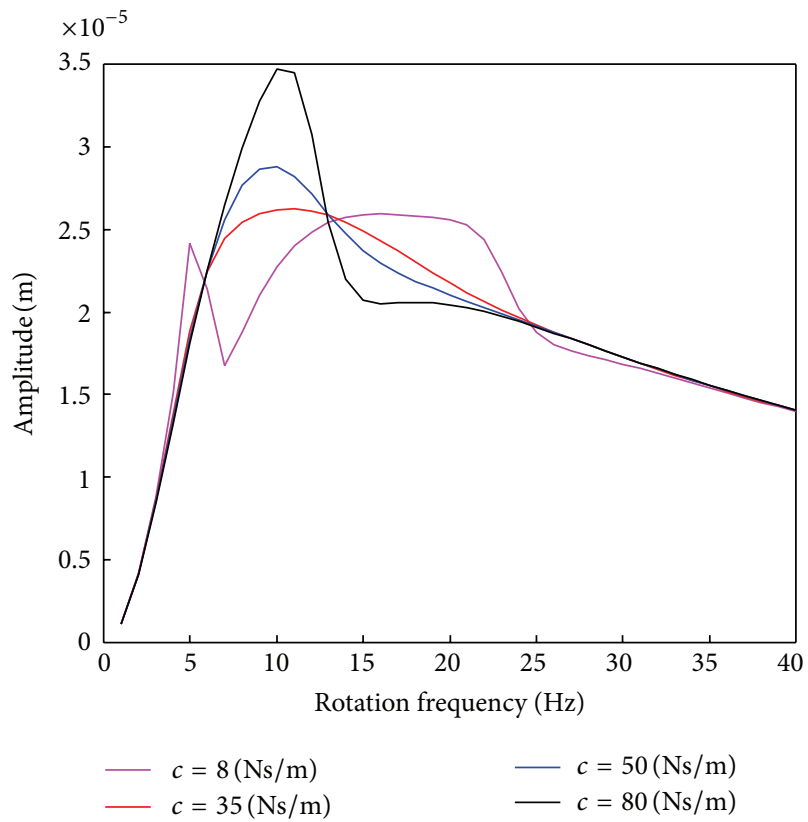

(d) Response of the lower damper

FIGURE 7: Optimization of the damping coefficient.

damper's mass, damper's stiffness $K_{t}$, and magnetic coupling stiffness $K_{m}$. But, in the practical application, the $\omega_{t}$ is a little higher than the frequency to be tuned

$$
\omega_{t}=\sqrt{\frac{K_{t}+K_{m}}{M_{t}}} .
$$

(2) We need to hunt for the public point through regulating the parameters of (17). Since it is difficult to regulate the stiffness and mass of the damper, we need to adjust the magnetic coupling stiffness to hunt for the public points. If the magnetic coupling stiffness cannot satisfy the conditions, we will regulate the stiffness and mass. The law to adjust is that the change to amplify the $\omega_{t}$ can enhance the public point at the lower frequency.

(3) After finding the best public point, we need to adjust the damping coefficient to coincide with the public point and one peak. The method to hunt for the best damp is that we select a bigger and smaller damping coefficient to calculate and hunt for an adaptable parameter between them. The regulating law is that amplifying the damping coefficient will move the peak at the lower frequency to the higher and one or two peaks will coincide with the public point.

Since the upper damper plays a powerful role in the lower order modal, we will select and optimize the parameter of the upper supporting system based on the avoiding vibration 
theory at the first and second order critical frequency of the rotor system. Therefore, it is necessary to optimize the damp coefficient and magnetic coupling stiffness, which is helpful to realize the characteristic and provide reference for the design.

Figure 6 displays the response of the rotor system's four nodes by selecting different magnetic coupling stiffness. If the magnetic coupling stiffness deviates from the best value along the direction of the decrease, the amplitude of all the nodes will increase greatly and if it deviates from the best value along the direction of the increase, the amplitude of the nodes will decrease, but the change is relatively small beside the top one. It is clear that increasing magnetic coupling stiffness is beneficial to pass through the critical speed, which can be explained by energy dissipation. If the magnetic coupling stiffness is infinite, the rotor and damper will become a unity and damper will support great damp. But, the value of the magnetic coupling stiffness is limited by other application conditions which will be introduced in another paper of the author.

After adopting an adaptable magnetic coupling stiffness, the response of the rotor system's four nodes is presented in Figure 6 when the upper damper selects different damp. Figure 7 displays the response curves of the upper damper, top rotor, middle rotor, and lower damper adopting different damping coefficient of the upper damper. We can conclude that there are two peaks at the lower damping coefficient and the two peaks will be merged into one which has higher amplitude at the higher damping coefficient. Therefore, the damping coefficient of the red line can be the adaptable value based on the current parameter of the upper and lower supporting system.

We can select and optimize the parameter of the bottom supporting mechanism by applying the same method after selecting the parameter of the upper mechanism. It is relatively easy to achieve the best parameters of the avoiding vibration system by repeating calculation several times adopting the above steps and following the above rules. In order to describe the unbalance response of the rotor system concretely, Figures 8 and 9 present the undamped system and optimized response, respectively. We can clearly find that the amplitude of the rotor system has been reduced significantly due to the function of the damper.

\section{The Running and Debugging Experiment of the Rotor System}

How to choose damper and regulate its damp and magnetic coupling stiffness $K_{m}$ is very important to vertical rotor system to achieve higher speed smoothly. Because of the limit of the practical technology, the damp of the damper cannot reach the precise optimized value and it is relatively difficult to regulate its parameters once confirming the structure. The value of the $K_{m}$ is related to the clearance between ring shaped permanent magnet and iron closely which is simple to adjust relatively; therefore, the rotor can satisfy the requirements in the running process mainly by regulating the clearance after installing the rotor system. In order to observe the vibration amplitude of the response in the running experiment, we

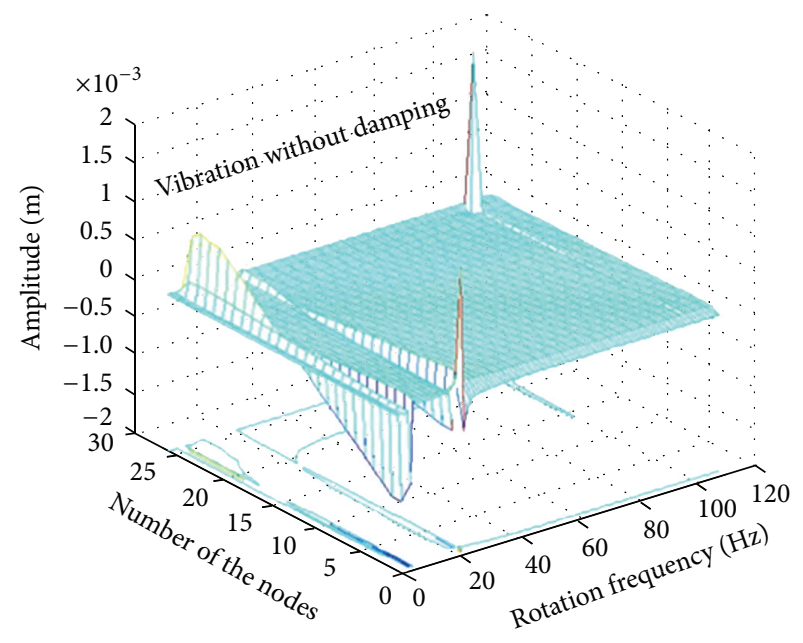

FiguRE 8: Response of the rotor system without damping.

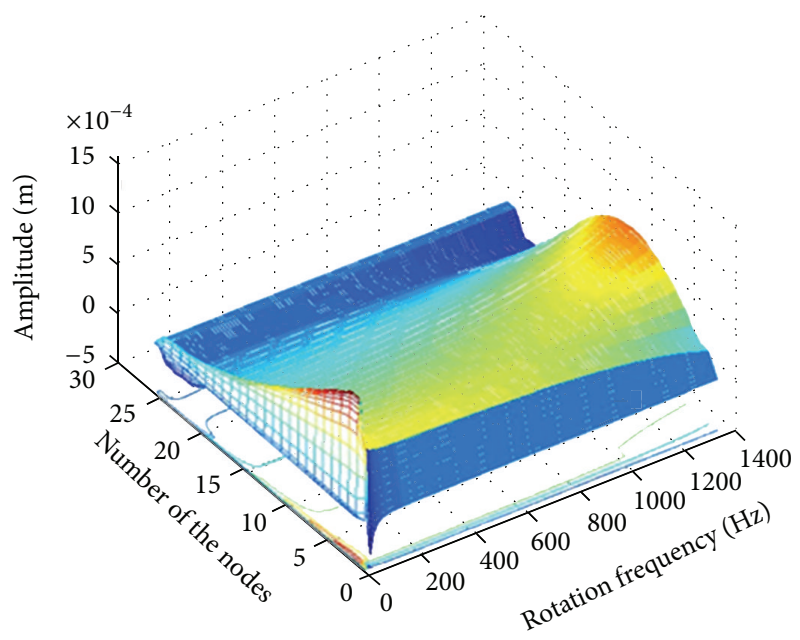

FIGURE 9: Response of the rotor system.

install two sensors in the top and middle of the rotor to achieve the response curves in the process of increasing speed, which will provide the reference for analyzing the vibration. We only extract the baseband signal from the vibration signal and regard it as the basis of theoretical analysis. There are also many factors to be concerned in the running experiment such as the vibration of the surrounding, vacuum of the environment, and angular acceleration of the speed. At last, the rotor reached $80000 \mathrm{r} / \mathrm{min}$ without any noise only after taking one day to debug. The response curve of the top and middle of the rotor is presented in Figure 10.

\section{Conclusion}

This paper introduces a supporting mechanism of the vertical rotor whose rotate speed can reach $80000 \mathrm{r} / \mathrm{min}$ and optimizes its parameters through applying the above optimization method, which significantly shortens the debugging time and improves the work efficiency. The optimization 


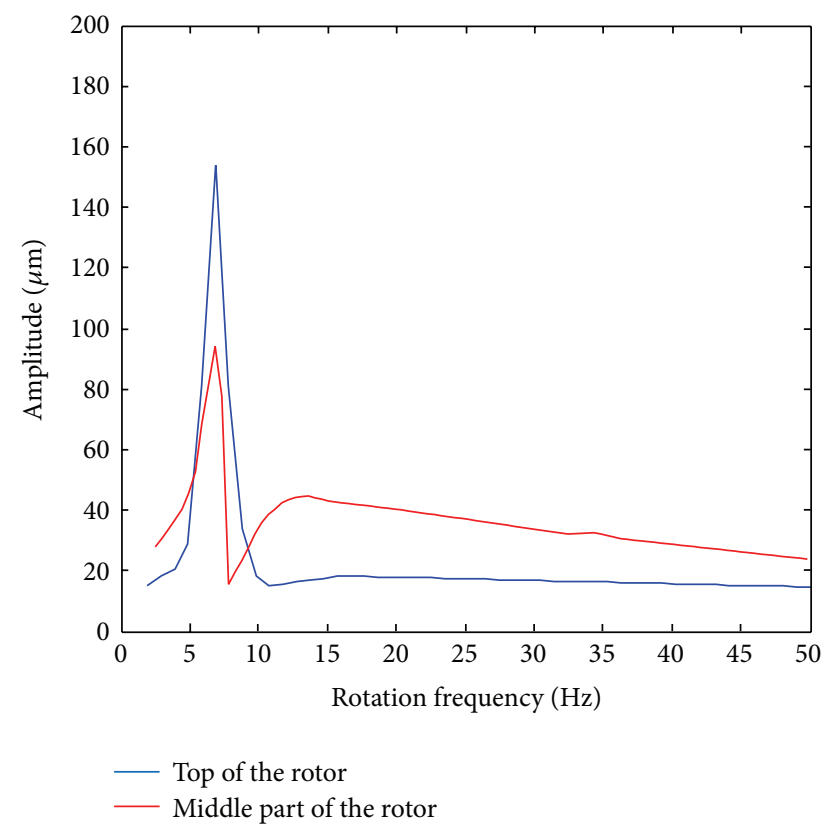

FIGURE 10: Response curves of the rotor.

results show that the vibration amplitude of the top supporting mechanism reduces from $350 \mu \mathrm{m}$ to $150 \mu \mathrm{m}$ and the amplitude of the middle supporting mechanism reduces from $210 \mu \mathrm{m}$ to $90 \mu \mathrm{m}$, which indicates that the rotor can rotate smoothly. Since the main influencing factors of vibration are found and the matching principle of the upper and bottom supporting mechanism's parameters is provided in this study, the design and debugging period is significantly shortened. There are four points that need to be introduced in the process of the optimization and experiment: (1) the FEM plays a powerful role in the dynamic analysis considering the gyroscopic couple; (2) the design and match of the upper and lower supporting mechanism in the avoiding vibration system of the rotor system are very important to pass the lower order critical speed smoothly; (3) the matching principle of the upper and bottom supporting system in the two-degree freedom system can also be applied to the multiple degree freedom system; (4) how to regulate the clearance between the ring permanent magnet and iron in the upper supporting mechanism is the heart in the running experiment.

\section{Conflict of Interests}

The authors declare that there is no conflict of interests regarding the publication of this paper.

\section{References}

[1] D.-K. Hong, B.-C. Woo, J.-Y. Lee, and D.-H. Koo, "Ultra high speed motor supported by air foil bearings for air blower cooling fuel cells," IEEE Transactions on Magnetics, vol. 48, no. 2, pp. 871-874, 2012.
[2] B. H. Ertas, M. Camatti, and G. Mariotti, "Synchronous response to rotor imbalance using a damped gas bearing," Journal of Engineering for Gas Turbines and Power, vol. 132, no. 3, Article ID 032501, 2010.

[3] K. Hikichi, K. Shiratori, S. Togo, and K. Hioka, "Hydroinertia gas bearings and their application to high speed micro spinners," Journal of Japanese Society of Tribologists, vol. 50, no. 6, pp. 465-470, 2005.

[4] A. Palazzolo, "Magnetic bearing development for support of satellite flywheels," in Space technology and applications international forum, vol. 420 of AIP Conference Proceedings, pp. 685692, 1998.

[5] C. DellaCorte, "Oil-Free shaft support system rotordynamics: past, present and future challenges and opportunities," Mechanical Systems and Signal Processing, vol. 29, pp. 67-76, 2012.

[6] A. Tonoli, N. Amati, A. Bonfitto, M. Silvagni, B. Staples, and E. Karpenko, "Design of electromagnetic dampers for aero-engine applications," Journal of Engineering for Gas Turbines and Power, vol. 132, no. 11, Article ID 112501, pp. 699-710, 2010.

[7] J. Mahfoud and J. Der Hagopian, "Investigations on critical speed suppressing by using electromagnetic actuators," Smart Structures and Systems, vol. 9, no. 4, pp. 303-311, 2012.

[8] J. Zapoměl and P. Ferfecki, "A computational investigation on the reducing lateral vibration of rotors with rolling-element bearings passing through critical speeds by means of tuning the stiffness of the system supports," Mechanism and Machine Theory, vol. 46, no. 5, pp. 707-724, 2011.

[9] Y. Ishida, "Recent development of the passive vibration control method," Mechanical Systems and Signal Processing, vol. 29, pp. 2-18, 2012.

[10] F. J. Doubrawa Filho, M. A. Luersen, and C. A. Bavastri, "Optimal design of viscoelastic vibration absorbers for rotating systems," Journal of Vibration and Control, vol. 17, no. 5, pp. 699710, 2011.

[11] D.-K. Hong, B.-C. Woo, and D.-H. Koo, "Rotordynamics of 120 $000 \mathrm{r} / \mathrm{min} 15 \mathrm{~kW}$ ultra high speed motor," IEEE Transactions on Magnetics, vol. 45, no. 6, pp. 2831-2834, 2009.

[12] T. Majewskia, "Automatic elimination of vibrations for a centrifuge," Mechanism and Machine Theory, vol. 46, no. 3, pp. 344357, 2011.

[13] J. Chung and I. Jang, "Dynamic response and stability analysis of an automatic ball balancer for a flexible rotor," Journal of Sound and Vibration, vol. 259, no. 1, pp. 31-43, 2003.

[14] C. Rajalingham, R. B. Bhat, and S. Rakheja, "Automatic balancing of flexible vertical rotors using a guided ball," International Journal of Mechanical Sciences, vol. 40, no. 9, pp. 825-834, 1998.

[15] T. Majewski, "Automatic balancing of a rotor in two planes," Archives of Mechanics, vol. 27, no. 2, pp. 193-212, 1980.

[16] H. Taplak and M. Parlak, "Evaluation of gas turbine rotor dynamic analysis using the finite element method," Measurement, vol. 45, no. 5, pp. 1089-1097, 2012.

[17] Y. S. Chen, Y. D. Cheng, J. J. Liao, and C. C. Chiou, "Development of a finite element solution module for the analysis of the dynamic behavior and balancing effects of an induction motor system," Finite Elements in Analysis and Design, vol. 44, no. 8, pp. 483-492, 2008.

[18] F. Strauß, V. Heuveline, and B. Schweizer, "Existence and approximation results for shape optimization problems in rotordynamics," Numerische Mathematik, vol. 109, no. 2, pp. 311$332,2008$. 
[19] H.-T. Lim, W.-B. Jeong, and K.-J. Kim, "Dynamic modeling and analysis of drum-type washing machine," International Journal of Precision Engineering and Manufacturing, vol. 11, no. 3, pp. 407-417, 2010.

[20] Z. Yuan, F. Chu, and R. Hao, "Simulation of rotor's axial rubimpact in full degrees of freedom," Mechanism and Machine Theory, vol. 42, no. 7, pp. 763-775, 2007.

[21] E. Pedersen, "Rotordynamics and bond graphs: basic models," Mathematical and Computer Modelling of Dynamcial Systems: Methods, Tools and Applications in Engineering and Related Sciences, vol. 15, no. 4, pp. 337-352, 2009.

[22] U. Werner, "Elastic multiple-mass model for rotordynamic analysis of flexible electrical rotors," Engineering Research, vol. 75, no. 4, pp. 209-229, 2011.

[23] Z. Yie, Rotordynamics, Tsinghua University Press, Beijing, China, 1987.

[24] B.-G. Liu, "Eigenvalue problems of rotor system with uncertain parameters," Journal of Mechanical Science and Technology, vol. 26, no. 1, pp. 1-10, 2012. 


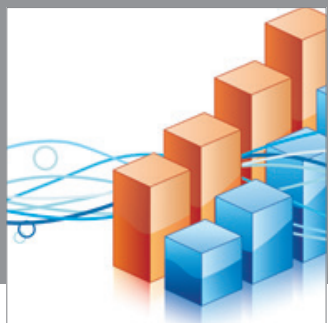

Advances in

Operations Research

mansans

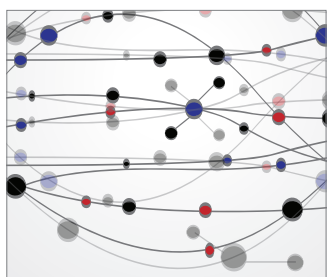

The Scientific World Journal
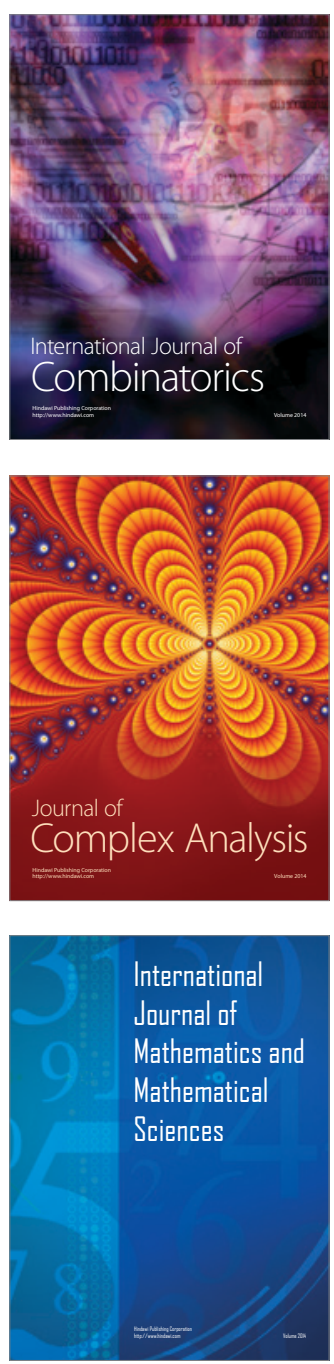
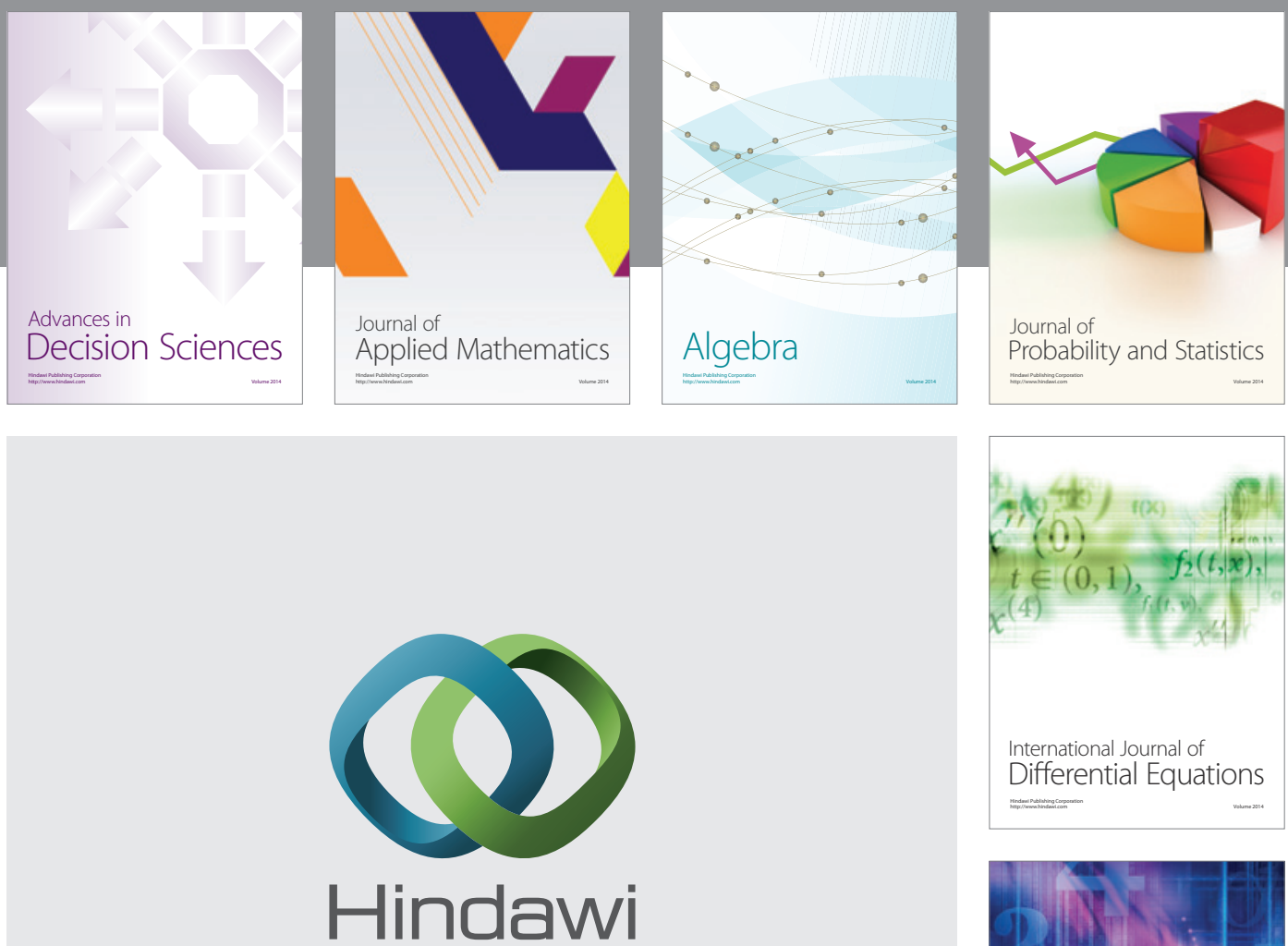

Submit your manuscripts at http://www.hindawi.com
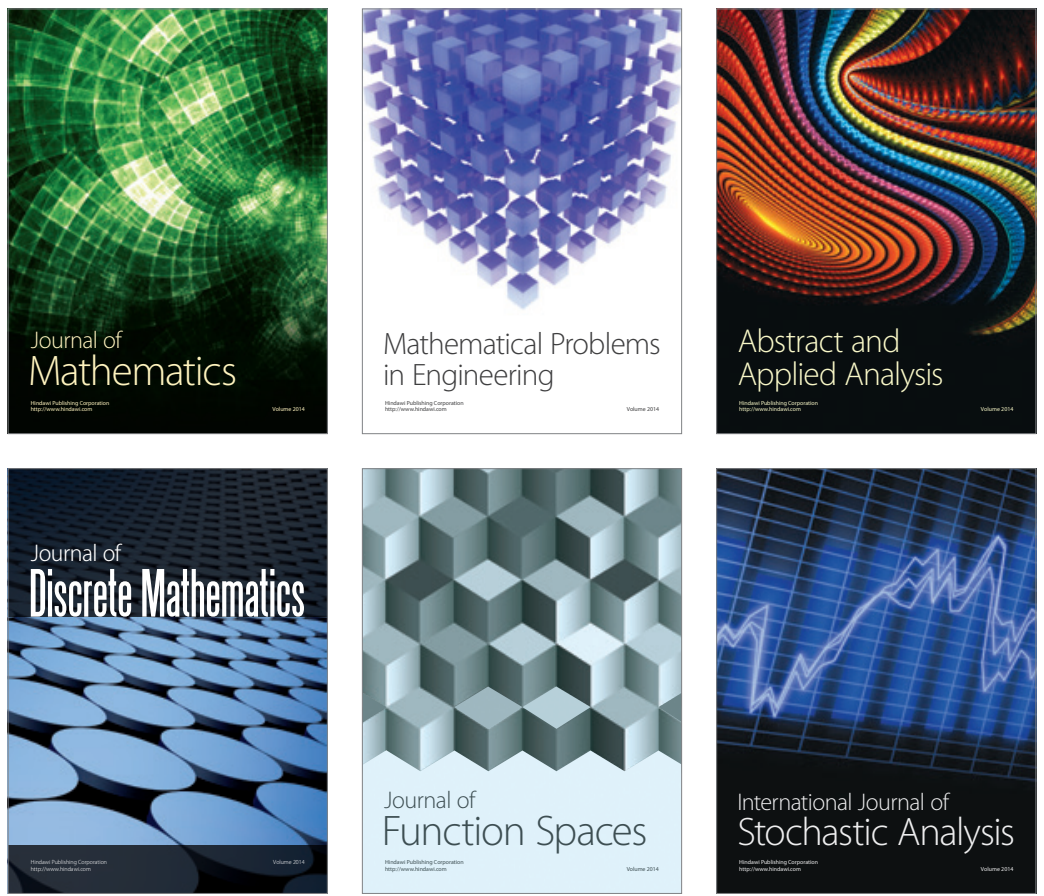

Journal of

Function Spaces

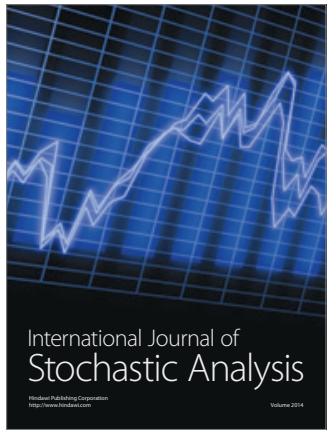

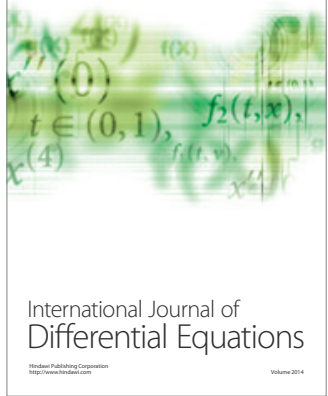
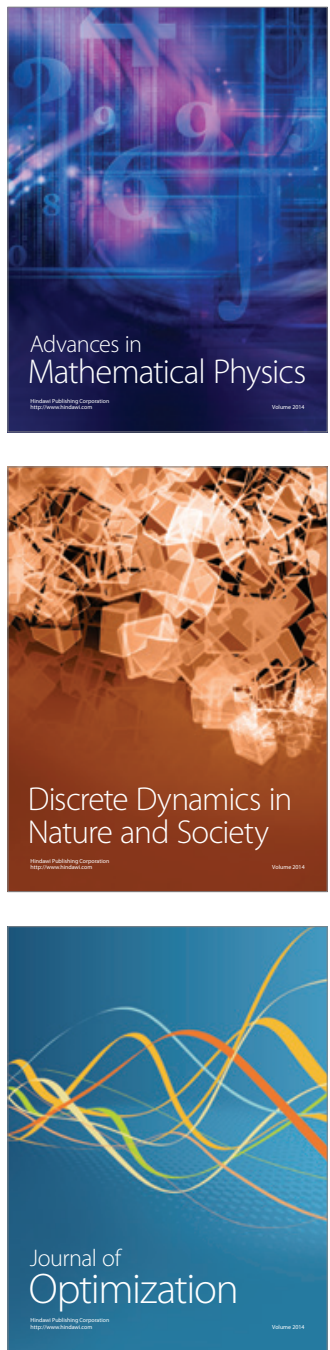